Dilani Wanasinghe, Shetal Shah, Amruta Bamanikar, David Aboudi, Soumya Mikkilineni, Olivia Shyong, Natasha Wu, Tessa Lavan and Heather L. Brumberg*

\title{
Lifestyle characteristics of parental electronic cigarette and marijuana users: healthy or not?
}

https://doi.org/10.1515/jpm-2019-0392

Received October 23, 2019; accepted November 2, 2019; previously published online December 19, 2019

\section{Abstract}

Objective: To assess lifestyle characteristics among parental electronic cigarette (e-cigarette), marijuana and tobacco users.

Methods: A total of 1214 parents (77\% mothers and $23 \%$ fathers) were surveyed and categorized into five exposure groups: e-cigarette use only (1\%), marijuana use only (3\%), tobacco products only (10\%), multi-exposed [11\% (marijuana, e-cigarette and tobacco)], and non-users [75\% (no e-cigarette, tobacco or marijuana)].

Results: Similar to non-users, the e-cigarette group had no illicit drug use. Further, e-cigarette users were more likely, in adjusted models, to self-identify as non-smokers and exercise compared with tobacco and multi-exposed groups. Although marijuana users also had higher odds of self-identifying as non-smokers compared to tobacco and multi-exposed groups, they were more likely than nonusers to drink alcohol.

Conclusion: E-cigarette and marijuana using parents were less likely to identify as smokers. E-cigarette users had healthier lifestyle characteristics than the other exposed

*Corresponding author: Heather L. Brumberg, MD, MPH, FAAP, Department of Pediatrics, Division of Newborn Medicine, Maria Fareri Children's Hospital at Westchester Medical Center, New York Medical College, 100 Woods Road, Valhalla, NY 10595, USA; and The Regional Neonatal Center, Division of Newborn Medicine, New York Medical College, Maria Fareri Children's Hospital at Westchester Medical Center, Valhalla, NY, USA, Phone: +(914) 493-8491, Fax: +(914) 493-1005,

E-mail: heather_brumberg@nymc.edu

Dilani Wanasinghe, Shetal Shah and David Aboudi: The Regional Neonatal Center, Division of Newborn Medicine, New York Medical College, Maria Fareri Children's Hospital at Westchester Medical Center, Valhalla, NY, USA

Amruta Bamanikar: Jersey Shore University Medical Center - a University Level Affiliate of Rutgers Robert Wood Johnson, Neptune, NJ, USA

Soumya Mikkilineni and Olivia Shyong: Westchester Medical Center, New York Medical College, Valhalla, NY, USA

Natasha Wu: Geisinger School of Medicine, Wilkes-Barre, PA, USA

Tessa Lavan: Hamilton College, Clinton, NY, USA groups. Clinicians should consider specifically screening for parental e-cigarette and marijuana use as assessing for only "smoking" may underrepresent first-and secondhand exposure. Additionally, clinicians should be aware that marijuana using parents are more likely to drink alcohol and should counsel accordingly.

Keywords: behaviors; e-cigarettes; lifestyle; marijuana; parents.

\section{Introduction}

Rates of combustible tobacco use have been declining over the past several decades [1]. Yet electronic cigarette (e-cigarette) use is increasing in adults $\geq 25$ years, having doubled to 4.2\% between 2012 and 2014 [1]. Among e-cigarette users, $11.4 \%$ vape exclusively, having never smoked tobacco [2]. In contrast, $60 \%$ of e-cigarette users supplement vaping with traditional cigarette use [2]. Furthermore, exclusive e-cigarette users are at increased risk for initiating subsequent combustible cigarette use within 2 years [3-7]. Almost a third of women in Oklahoma and Texas who use both e-cigarettes and combustible tobacco report using both products in the preconception period [8]. Further, $6 \%$ of non-smokers vaped $>3$ months before pregnancy. However, this rate was reduced to $0.5 \%$ during the last 3 months of pregnancy [8].

Though perceived as safer than traditional cigarettes [9], e-cigarettes contain potentially toxic inhalants such as formaldehyde, acetaldehyde and acrolein [10]. As a result, oxidative stress and inflammatory responses in the lung and irritation throughout the respiratory tract are potential consequences of e-cigarette use [11]. The chemicals present in e-cigarette aerosols are mutagenic and may, in users, increase risk of cancer and adverse reproductive outcomes [10]. Several of these substances cross the placenta and may impact the developing fetus [12]. In animal models, exposure to e-cigarette vapor during pregnancy is linked with both greater maternal and neonatal inflammatory markers [13]. Although there are no human studies, there is a higher risk of decreased body weight, impaired postnatal lung growth, and impaired cardiac function for offspring born to pregnant animals exposed to e-cigarette vapor 
[14]. Animal models have also demonstrated an association between maternal e-cigarette exposure with long-term cognitive and epigenetic effects in offspring [15].

Similar to rates of e-cigarette use, marijuana use is highest among adults of reproductive age [16]. Marijuana and traditional cigarette use occur in up to $21 \%$ of young adults [17]. In contrast to alcohol and combustible tobacco consumption, marijuana use is increasing among pregnant women coincident with state-based legalization [18, 19]. Rates of marijuana use may be further underestimated based on self-report alone as Young-Wolff et al. [20] found that prevalence of marijuana use during pregnancy was higher based on toxicology than by patient recall [20]. Use among parents with children less than 18 years of age has also risen from $4.9 \%$ in 2002 to $6.8 \%$ in 2015 , increasing risk for childhood marijuana exposure [21].

Tetrahydrocannabinol is absorbed through the lungs and rapidly enters the blood stream reaching the brain within minutes [22]. Heavy, chronic use in young people can lead to long-term impairment of attention and memory which worsen with persistent use and early initiation [22]. Combustible marijuana has similarly harmful constituents as tobacco smoke [23]. Although evidence suggests that the risk of lung cancer is lower than with tobacco, marijuana smoking is also associated with adverse lung health such as inflammation of large airways, lung hyperinflation and chronic bronchitis [22].

Tetrahydrocannabinol rapidly crosses the placenta enabling concentrations in fetal blood to closely resemble those in maternal blood [24]. Thus, the fetus has an increased risk of exposure to marijuana and resultant adverse birth outcomes. Pregnant women using marijuana exclusively had 12 times greater odds of miscarriage or stillbirth as compared with non-users [25]. Additionally, marijuana use during pregnancy is associated with preterm labor, preterm birth, and decreased birth weight $[24,26]$. Infants with prenatal marijuana exposure are more likely to be admitted to the neonatal intensive care unit [26, 27]. Maternal marijuana use has been linked with long-term outcomes in children such as poor cognitive performance and increase behavioral problems such as depressive symptoms, anxiety and inattentiveness. However, there is currently insufficient evidence to establish causality [24, 28-30].

Despite these toxicities, pregnant women and adults of reproductive age perceived marijuana and e-cigarettes as safer than combustible tobacco $[8,31-36]$. Rates of both e-cigarette and marijuana use are increased with perception of decreased harm [37, 38]. Parental utilization of other substances with adverse health consequences such as illicit drugs and tobacco are associated with less healthy behaviors including decreased exercise [39, 40]. However, there is a paucity of data on the associated lifestyle behaviors of marijuana and e-cigarette users, particularly among parents of newborns. Therefore, we sought to assess lifestyle characteristics, including identification as a non-smoker, exercise, alcohol use and illicit drug use, in relation to parental use of e-cigarettes and marijuana.

\section{Materials and methods}

This is an analysis of self-administered survey data collected from parents of live-born infants at Maria Fareri Children's Hospital (MFCH) at Westchester Medical Center, Valhalla, NY, USA and six affiliate birthing hospitals in the Hudson Valley Region of New York. Inclusion criteria were parents $\geq 18$ years of age, with singleton live births, who speak Spanish or English. Parents were excluded if they spoke a language other than Spanish or English, were $<18$ years of age, did not have the capacity to consent, or had multiple births. Surveys were administered between July 2016 and July 2018. The study was approved by the NY Medical College Institutional Review Board for the Protection of Human Subjects. We have complied with the World Medical Association Declaration of Helsinki regarding ethical conduct of research involving human subjects.

\section{Data collection}

Survey tools: Maternal and paternal survey tools (Supplementary Material) were adapted from the Center of Disease Control's Pregnancy Risk Assessment Monitoring System-Phase 7 [41], the National Institutes of Health and Food and Drug Administration collaborative study - Population Assessment of Tobacco and Health [42], the National Health and Nutrition Examination Survey [43] and the U.S. Census Bureau [44]. The validated survey included 39 questions in the maternal survey and 37 in the paternal questionnaire. Maternal surveys differed from paternal by a question on prenatal care and smoking cessation during pregnancy.

\section{Variable definitions}

Exposure groups included exclusive e-cigarette users, exclusive marijuana users [marijuana, pot, bud, hashish, K2 (synthetic marijuana)], tobacco product users (hookah, smokeless tobacco, snus pouches, cigarettes and cigars), multi-exposed (two or more of e-cigarette, marijuana or tobacco products), and non-users (absence of e-cigarette, tobacco or marijuana products). E-cigarette use was defined as use 12 months prior to or during the pregnancy [42]. Tobacco use was defined as use 3 months prior to or during pregnancy [42]. Marijuana use was defined as use 1 month prior to or during pregnancy [41].

Lifestyle behaviors include physical activity, identification as a non-smoker, alcohol use and substance use. Physical activity was defined as exercise $\geq 3$ days per week or $<3$ days per week during the 12 months prior to the pregnancy [41]. Alcohol use was defined as any use 3 months prior to or during the pregnancy [41]. Illicit drug 
use included use of cocaine, amphetamines or tranquilizers 1 month prior to or during pregnancy [42].

Covariates included demographics such as age, education, marital status, employment, race/ethnicity and adverse life events. Education was defined as less than or at least completion of high school or greater (includes some college or associate's degree, Bachelor's degree and advanced degree) [35, 42, 45]. Subjects were considered married if they self-reported being married or in a domestic partnership and not married if separated, single, never married, widowed or divorced [43, 46]. Adverse life events were defined by 14 factors including homelessness, trouble paying rent or mortgage or other bills, family sickness, partner incarceration, unwanted pregnancy, divorce, military deployment, partner separation, partner discord, the death of a close relative/friend, the presence of excessive alcohol or drug use in a close family member/friend and reduced or loss of employment [41]. Adverse life events score included the total number of factors reported and was categorized into those reporting $\geq 3$ events and $<3$ events [47].

\section{Statistical analysis}

Lifestyle behaviors, covariates and demographics were compared by e-cigarette use, marijuana use, tobacco use, multi-exposed and nonuse in bivariate analysis using chi-square $\left(\chi^{2}\right)$ or Fisher's exact tests. Multiple logistic regression models were used to determine odds ratios and 95\% confidence intervals (CI) for the association of parental exposures and lifestyle behaviors while controlling for covariates. These covariates were selected if they had a P-value of 0.1 or less in the bivariate analysis. Final models controlled for race/ethnicity, age in years, gender, education and adverse life events score. Separate models were conducted using exclusive e-cigarette users and exclusive marijuana users as reference categories. Due to paucity of illicit drug use, multivariate analysis of this behavior could not be conducted. Receiver operator characteristic (ROC) curves with concordance index (C-statistic) were generated to assess predictive accuracy modeling. Statistical significance was determined by a P value less than 0.05. Analyses utilized STATA version 14.0 (Stata Corporation, College Station, TX, USA).

\section{Results}

A total of 1689 mothers and 811 fathers were approached of which 969 mothers and 297 fathers consented (Figure 1). Fifty-two were excluded, two of which withdrew consent and 50 had incomplete consent forms. A total of 1214 subjects were included in the analysis. The maternal response rate was 57\% and paternal response rate was 37\%. There were $1 \%$ e-cigarette users, $3 \%$ marijuana users, $10 \%$ tobacco product users, $11 \%$ multi-exposed and $75 \%$ non-users.

\section{Bivariate analysis}

Table 1 shows the characteristics among exposure groups (e-cigarette, marijuana, tobacco, multi-exposed and nonusers). Tobacco users and non-users were on average older than e-cigarette users, marijuana users and multiexposed. The multi-exposed had fewer Hispanic and more White parents compared with other groups. Mothers were more likely to be e-cigarette users or non-users compared to fathers. E-cigarette users were least likely to have attained education beyond high school, and non-users were more often married. The multi-exposed had the highest rate of adverse life events. Employment status was similar among all groups.

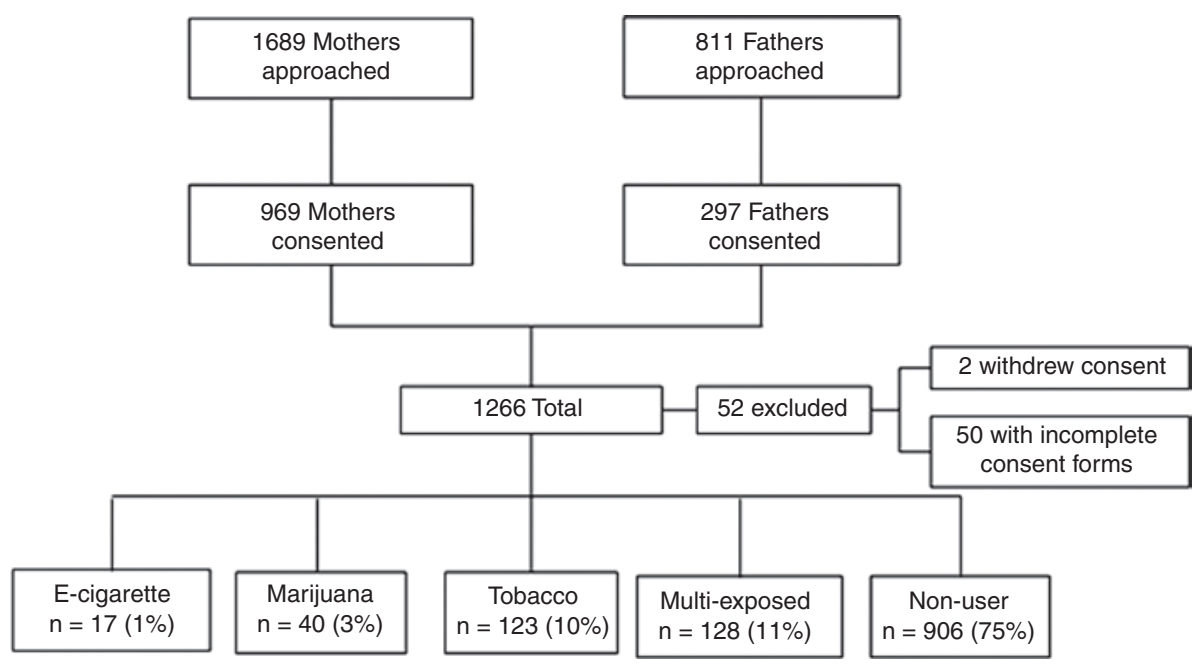

Figure 1: Flow diagram of subjects approached, consented, and included by exposure group.

E-cigarette, e-cigarette use only; marijuana, marijuana use only; tobacco, tobacco product use only; multi-exposed, any combination of e-cigarette, marijuana or tobacco products; non-user, neither e-cigarette, marijuana, nor tobacco use. 
Table 1: Demographics and characteristics by exposure groups.

\begin{tabular}{|c|c|c|c|c|c|c|c|}
\hline \multicolumn{2}{|l|}{ n (\%) } & \multirow{2}{*}{$\frac{\text { E-cigarette } \mathbf{n}=17}{5(29)}$} & \multirow{2}{*}{$\frac{\text { Marijuana } n=40}{14(35)}$} & \multirow{2}{*}{$\begin{array}{r}\text { Tobacco } \mathbf{n}=123 \\
26(21)\end{array}$} & \multirow{2}{*}{$\begin{array}{r}\text { Multi-exposed } \mathbf{n}=\mathbf{1 2 8} \\
38(30)\end{array}$} & \multirow{2}{*}{$\begin{array}{r}\text { Non-user } \mathbf{n}=906 \\
143(16)\end{array}$} & \multirow{2}{*}{$\frac{\text { P-value }}{<0.001}$} \\
\hline Age, years & $18-24$ & & & & & & \\
\hline & $25-35$ & $9(53)$ & $19(48)$ & $70(57)$ & $68(53)$ & $504(56)$ & \\
\hline & $>35$ & $3(18)$ & $7(18)$ & $27(22)$ & $22(17)$ & $249(28)$ & \\
\hline \multirow[t]{2}{*}{ Gender } & Male & $3(8)$ & $14(35)$ & $43(35)$ & $46(36)$ & $173(19)$ & $<0.001$ \\
\hline & Female & $14(82)$ & $26(65)$ & $80(65)$ & $82(64)$ & $733(81)$ & \\
\hline \multirow[t]{4}{*}{ Race } & White & $8(47)$ & $15(38)$ & $51(44)$ & $70(57)$ & $338(40)$ & 0.008 \\
\hline & Black & $3(18)$ & $8(21)$ & $20(17)$ & $25(20)$ & $134(16)$ & \\
\hline & Hispanic & $6(35)$ & $16(41)$ & $41(35)$ & $25(20)$ & $327(38)$ & \\
\hline & Other & $0(0)$ & $0(0)$ & $5(4)$ & $3(2)$ & $56(7)$ & \\
\hline \multicolumn{2}{|c|}{$>$ High school $^{\mathrm{a}}$} & $6(35)$ & $21(54)$ & $65(53)$ & $59(47)$ & $579(65)$ & $<0.001$ \\
\hline \multicolumn{2}{|l|}{ Employed } & $11(65)$ & $24(60)$ & $74(61)$ & $76(59)$ & $572(64)$ & 0.797 \\
\hline \multicolumn{2}{|l|}{ Married } & $11(65)$ & $17(51)$ & $57(52)$ & $45(41)$ & $632(72)$ & $<0.001$ \\
\hline \multicolumn{2}{|c|}{ Adverse life events ${ }^{b}$} & $1(6)$ & $5(13)$ & $29(24)$ & $57(45)$ & $95(10)$ & $<0.001$ \\
\hline \multicolumn{2}{|c|}{ Identify as non-smoker } & $12(71)$ & $26(65)$ & $32(26)$ & $11(9)$ & $869(96)$ & $<0.001$ \\
\hline \multicolumn{2}{|c|}{ Exercise for $\geq 3$ days } & $11(65)$ & $17(43)$ & $47(39)$ & $45(36)$ & $392(44)$ & 0.111 \\
\hline \multicolumn{2}{|l|}{ Alcohol $^{c}$} & $8(47)$ & $29(73)$ & $83(67)$ & $87(68)$ & $430(47)$ & $<0.001$ \\
\hline \multicolumn{2}{|c|}{ Illicit drug use $\mathrm{d}^{\mathrm{d}}$} & $0(0)$ & $1(3)$ & $1(1)$ & $14(11)$ & $0(0)$ & $<0.001$ \\
\hline
\end{tabular}

a More than completion of high school. bScore including $\geq 3$ events. 'Use 3 months prior to or during pregnancy. dUse 1 month prior to or during pregnancy. E-cigarette, e-cigarette use only; marijuana, marijuana use only; tobacco, tobacco product use only; multi-exposed, any combination of e-cigarette, marijuana or tobacco products; non-user, neither e-cigarette, marijuana, nor tobacco use. Bold designates $\mathrm{P}<0.05$.

E-cigarette users and marijuana users were more likely to identify as non-smokers than tobacco users and the multi-exposed. Non-users were most likely to report non-smoking status. Groups did not differ in rates of exercise. E-cigarette users and non-users had the lowest rates of alcohol use while marijuana users had the highest. The multi-exposed had the highest rate of illicit drug use. There was no illicit drug use in the e-cigarette and non-user groups precluding further multivariate analysis.

\section{Multivariate analysis}

Table 2 shows results of multivariate models using e-cigarette users and marijuana users as reference groups and controlling for age, race/ethnicity, gender, education and adverse life events. Other than non-users, e-cigarette users exhibited the healthiest lifestyle characteristics, including higher odds of identifying as a non-smoker and increased odds of exercise compared to tobacco users and the multi-exposed. Although, marijuana users were more likely than tobacco users and the multi-exposed to identify as non-smokers, they were more likely than non-users to consume alcohol.

\section{Discussion}

Our study is the first to assess the lifestyle characteristics of exclusive parental e-cigarette and marijuana users. As expected, non-users had the healthiest lifestyle characteristics including identification as a non-smoker, decreased alcohol use and no illicit drug use. Interestingly, exclusive e-cigarette users overwhelmingly identified as non-smokers and exhibited other healthier choices. While marijuana users also identified as a non-smoker, they were more likely to use alcohol.

Parental use of tobacco and illicit substances correlate with unhealthy behaviors [39, 40]. In our study, exclusive e-cigarette and marijuana use was associated with healthier characteristics. Our findings suggest parents who engage in healthy activities such as exercise may be similarly choosing substances they perceive as healthier alternatives to tobacco and illicit drugs. Absence of widespread e-cigarette regulations including graphic label warnings and child-appealing flavoring may amplify the false belief of safety [10]. Furthermore, state-based legalization of marijuana also contributes to an increased perception of safety [48].

Interestingly, both e-cigarette users and marijuana users did not identify as smokers. Self-identification as a 
Table 2: Multivariate logistic regression comparing behaviors among groups to e-cigarette and marijuana.

\begin{tabular}{|c|c|c|c|c|c|}
\hline \multicolumn{2}{|c|}{ Outcome: lifestyle behaviors ${ }^{\mathrm{a}}$} & \multicolumn{2}{|l|}{ Marijuana $^{\mathrm{b}}$} & \multicolumn{2}{|l|}{ E-cigarette ${ }^{c}$} \\
\hline & & OR $(95 \% \mathrm{Cl})$ & C-statistic & OR $(95 \% \mathrm{Cl})$ & C-statistic \\
\hline \multirow[t]{5}{*}{ Not exercise $\geq 3$ days } & E-cigarette & $0.35(0.1-1.2)$ & 0.62 & 1 (ref) & 0.62 \\
\hline & Marijuana & 1 (ref) & & $2.86(0.9-9.6)$ & \\
\hline & Tobacco & $1.19(0.6-2.6)$ & & $3.34(1.1-10.0)$ & \\
\hline & Multi-exposed & $1.32(0.6-2.9)$ & & $3.78(1.3-11.2)$ & \\
\hline & Non-user & $0.95(0.5-1.9)$ & & $2.71(0.97-7.6)$ & \\
\hline \multirow[t]{5}{*}{ Alcohold $^{\mathrm{d}}$} & E-cigarette & $0.33(0.1-1.2)$ & 0.74 & 1 (ref) & 0.74 \\
\hline & Marijuana & 1 (ref) & & $3.01(0.8-11.0)$ & \\
\hline & Tobacco & $0.78(0.3-1.9)$ & & $2.35(0.8-7.2)$ & \\
\hline & Multi-exposed & $0.74(0.3-1.8)$ & & $2.24(0.7-6.9)$ & \\
\hline & Non-user & $0.29(0.1-0.7)$ & & $0.89(0.3-2.5)$ & \\
\hline \multirow[t]{5}{*}{ Identify as a smoker } & E-cigarette & $0.63(0.2-2.2)$ & 0.91 & 1 (ref) & 0.91 \\
\hline & Marijuana & 1 (ref) & & $1.60(0.5-5.6)$ & \\
\hline & Tobacco & $4.57(2.1-10.1)$ & & $7.31(2.3-23.1)$ & \\
\hline & Multi-exposed & $16.38(6.4-41.7)$ & & $26.19(7.4-92.1)$ & \\
\hline & Non-user & $0.07(0.03-0.2)$ & & $0.11(0.04-0.3)$ & \\
\hline
\end{tabular}

${ }^{\mathrm{a}}$ Adjusting for race/ethnicity, age, gender, education, and adverse life events score. ${ }^{\mathrm{b}}$ Marijuana is a reference category. ${ }^{\mathrm{C}} \mathrm{E}$-cigarette is a reference category. ${ }^{\mathrm{d} A l c o h o l ~ u s e ~} 3$ months prior to or during pregnancy. Bold designates $\mathrm{P}<0.05$. E-cigarette, e-cigarette use only; marijuana, marijuana use only; tobacco, tobacco product use only, multi-exposed, any combination of e-cigarette, marijuana or tobacco products; non-user, neither e-cigarette, marijuana, nor tobacco use.

non-smoker has not been well studied in parents. Our study is the first to assess identification as a non-smoker among multiple exposure groups and correctly predicted $91 \%$ of those identifying as non-smokers $(C$-statistic $=0.91)$. This finding has clinical implications highlighting the necessity for providers to specifically screen for e-cigarette and marijuana use. Simply screening for "smoking" may be insufficient and under-represent patterns of use. Currently the American College of Obstetrics and Gynecology guidelines note the increase in e-cigarette use, but recommended screening tools continue to emphasize combustible tobacco products [49]. Thus, parents who vape or use marijuana but do not self-identify as smokers may be undetected. Our study supports the American Academy of Pediatrics most recent recommendation to assess for all nicotine exposure, specifically including screening for e-cigarette use as well as independently assessing for marijuana use [50].

Our analysis was unique in that exposures were assessed among parents of newborns. The rate of parental e-cigarette use in our study (1\%) was lower than the parental rate (4\%) reported by Garbutt et al. [35]. The difference in the rate of e-cigarette use we found in our study may represent conservative use in new parents as studies have shown pregnant women are less likely to use substances [51, 52]. We found comparable rates of marijuana use $(3 \%)$ to nationally reported rates in pregnant women (3.9\%) [53]. The rate of parental tobacco use in our study
$(10 \%)$ was consistent with nationally reported rates of tobacco use during pregnancy (10.9\%) [54].

There are several limitations to our study. Data was collected via a survey, thus, behaviors are subject to underreporting compared to toxicology [20] and recall bias. However, self-reported behavior may more accurately reflect clinician encounters which inform counseling on substance use. Duration of exposure to e-cigarette, marijuana and tobacco differed based on the validated survey questions used [Population Assessment of Tobacco and Health (PATH), Pregnancy Risk Assessment Monitoring System (PRAMS)] [41, 42]. Nevertheless, these durations of exposure are commonly reported in other studies [8, 17]. Additionally, our survey tool did not assess motivation for e-cigarette use. Previous studies have demonstrated that pregnant women perceived e-cigarette use as safer than combustible tobacco [9, 32, 34]. It is therefore plausible that e-cigarette use by parents could be perceived by them as a mechanism to quit combustible tobacco. E-cigarettes as a vehicle for cessation has been evaluated in several studies with mixed results $[9,10,55,56]$. Future studies should investigate parental motivation for e-cigarette use.

Another limitation is our low parental response rate (paternal 37\% and maternal 57\%). Greater medical, social and educational demands are placed on new parents during the postpartum hospitalization which may limit capacity to respond to research surveys. However, our 
response rate did fall into the typical response rate range [57]. Rates of certain exposures were low, especially parental e-cigarette users. However, we found significant differences that persisted in adjusted models that were highly predictive ( $91 \%$ correctly predicted identification as non-smokers).

Our study has several strengths. Our ability to control for adverse life events which are known to be associated with both substance use [40] and lifestyle behaviors [58], improves the validity of our conclusions. Furthermore, by isolating exclusive e-cigarette, marijuana and tobacco use in comparison with multi-exposed and non-users allows for better discernment of the association of exposures and a spectrum of clinically relevant lifestyle behaviors. By focusing on the parents, we further delineate lifestyle behaviors in a previously under-studied population.

Our study demonstrates exclusive parental e-cigarette and marijuana users do not identify as smokers. We also determined that exclusive parental e-cigarette users had healthier lifestyle behaviors while marijuana users had increased alcohol use. These findings should raise awareness in clinicians encountering adults of reproductive age during the preconception, pregnancy and postpartum time periods allowing providers to better screen and counsel families. Educational initiatives should be considered to raise awareness of potential harms in the setting of increased legalization of marijuana and a dearth of regulations around e-cigarettes.

Acknowledgments: We would like to thank all the mothers and fathers who took the time to participate in this study. We also would like to thank all of the research participants, including medical students-Christopher Stoll, Daniel Schwab, Brian Hanyok, Brian Yost, Mayra Ramirez, Jennifer Kutt, Dana Greene, Sarah Spiegel, Amythis Soltani, Mohammed Alzoobaee, Janki Shah, Bianca Zapanta, Maliha Rahman, Bryony Lucas, Kyle Mobbs, Minette Finney, Reba Gillis and Paolo Velendia, and clinical research coordinator - Clare Giblin for contributing immensely to data collection and the staff at affiliate hospitals for their cooperation.

Author contributions: All the authors have accepted responsibility for the entire content of this submitted manuscript and approved submission.

Research funding: None declared.

Employment or leadership: None declared.

Honorarium: None declared.

Competing interests: The funding organization(s) played no role in the study design; in the collection, analysis, and interpretation of data; in the writing of the report; or in the decision to submit the report for publication.

\section{References}

1. U.S. Department of Health and Human Services. E-cigarette use among youth and young adults. A Report of the Surgeon General. Atlanta, GA: U.S. Department of Health and Human Services, Centers for Disease Control and Prevention, National Center for Chronic Disease Prevention and Health Promotion, Office on Smoking and Health; 2016.

2. QuickStats: Cigarette smoking status among current adult e-cigarette users, by age group- national health interview survey, United States, 2015. Morb Mortal Wkly Rep 2016;65:1177.

3. Wills TA, Knight R, Sargent JD, Gibbons FX, Pagano I, Williams RJ. Longitudinal study of e-cigarette use and onset of cigarette smoking among high school students in Hawaii. Tob Control 2017;26:34-9.

4. Primack BA, Soneji S, Stoolmiller M, Fine MJ, Sargent JD. Progression to traditional cigarette smoking after electronic cigarette use among US adolescents and young adults. JAMA Pediatr 2015;169:1018-23.

5. Barrington-Trimis JL, Urman R, Berhane K, Unger JB, Boley Cruz T, Pentz M, et al. E-cigarettes and future cigarette use. Pediatrics 2016;138:e20160379.

6. Bold KW, Kong G, Camenga DR, Simon P, Cavallo DA, Morean $\mathrm{ME}$, et al. Trajectories of e-cigarette and conventional cigarette use among youth. Pediatrics 2018;141:e20171832.

7. Leventhal AM, Strong DR, Kirkpatrick MG, Unger JB, Sussman $S$, Riggs NR, et al. Association of electronic cigarette use with initiation of combustible tobacco product smoking in early adolescence. J Am Med Assoc 2015;314:700-7.

8. Kapaya M, D’Angelo DV, Tong VT, England L, Ruffo N, Cox S, et al. Use of electronic vapor products before, during, and after pregnancy among women with a recent live birth - Oklahoma and Texas, 2015. Morb Mortal Wkly Rep 2019;68:189-94.

9. Mark KS, Farquhar B, Chisolm MS, Coleman-Cowger VH, Terplan M. Knowledge, attitudes, and practice of electronic cigarette use among pregnant women. J Addict Med 2015;9:266-72.

10. Eaton DL, Kwan LY, Stratton K, editors. National Academies of Sciences, Engineering, and Medicine; Health and Medicine Division; Board on Population Health and Public Health Practice; Committee on the review of the Health Effects of Electronic Nicotine Delivery Systems. Public Health Consequences. Public health consequences of e-cigarettes. Washington, DC: National Academies Press (US); 2018.

11. Kaur G, Muthumalage T, Rahman I. Mechanisms of toxicity and biomarkers of flavoring and flavor enhancing chemicals in emerging tobacco and non-tobacco products. Toxicol Lett 2018;288:143-55.

12. Pidoux G, Gerbaud P, Guibourdenche J, Therond P, Ferreira F, Simasotchi $C$, et al. Formaldehyde crosses the human placenta and affects human trophoblast differentiation and hormonal functions. PLoS One 2015;10:e0133506.

13. Chen H, Li G, Chan YL, Chapman DG, Sukjamnong S, Nguyen T, et al. Maternal e-cigarette exposure in mice alters DNA methylation and lung cytokine expression in offspring. Am J Respir Cell Mol Biol 2018;58:366-77.

14. Whittington JR, Simmons PM, Phillips AM, Gammill SK, Cen R, Magann EF, et al. The use of electronic cigarettes in pregnancy: a review of the literature. Obstet Gynecol Surv 2018;73:544-9. 
15. Nguyen T, Li GE, Chen H, Cranfield CG, McGrath KC, Gorrie CA. Maternal e-cigarette exposure results in cognitive and epigenetic alternations in offspring in a mouse model. Chem Res Toxicol 2018;31:601-11.

16. Substance Abuse and Mental Health Services Administration. Results from the 2013 National Survey on Drug Use and Health: Summary of National Findings, NSDUH Series H-48, HHS Publication No. (SMA) 14-4863. Rockville, MD: Substance Abuse and Mental Health Services Administration; 2014.

17. Cohn AM, Abudayyeh H, Perreras L, Peters EN. Patterns and correlates of the co-use of marijuana with any tobacco and individual tobacco products in young adults from Wave 2 of the PATH Study. Addict Behav 2018;92:122-7.

18. Agrawal A, Rogers CE, Lessov-Schlaggar CN, Carter EB, Lenze SN, Grucza RA. Alcohol, cigarette, and cannabis use between 2002 and 2016 in pregnant women from a nationally representative sample. JAMA Pediatr 2019;173:95-6.

19. Ericksen K, Shah S, Brumberg HL. Public health implications of rising marijuana use in pregnancy in an age of increasing legalization. JAMA Pediatr 2019;173:606-7.

20. Young-Wolff KC, Tucker L, Alexeeff S, Armstrong MA, Conway $A$, Weisner $C$, et al. Trends in self-reported and biochemically tested marijuana use among pregnant females in California from 2009-2016. J Am Med Assoc 2017;318:2490-1.

21. Goodwin RD, Cheslack-Postava K, Santoscoy S, Bakoyiannis N, Hasin DS, Collins BN, et al. Trends in cannabis and cigarette use among parents with children at home: 2002 to 2015 . Pediatrics 2018;141:e20173506.

22. Volkow ND, Compton WM, Weiss SR. Adverse health effects of marijuana use. N Engl J Med 2014;371:879.

23. National Academies of Sciences, Engineering, and Medicine. The health effects of cannabis and cannabinoids: current state of evidence and recommendations for research. Washington, DC: The National Academies Press; 2017.

24. Thompson R, DeJong K, Lo J. Marijuana use in pregnancy: a review. Obstet Gynecol Surv 2019;74:415-28.

25. Coleman-Cowger VH, Oga EA, Peters EN, Mark K. Prevalence and associated birth outcomes of co-use of cannabis and tobacco cigarettes during pregnancy. Neurotoxicol Teratol 2018;68:84-90.

26. Gunn JK, Rosales CB, Center KE, Nuñez A, Gibson SJ, Christ C, et al. Prenatal exposure to cannabis and maternal and child health outcomes: a systematic review and meta-analysis. $\mathrm{Br}$ Med J Open 2016;6:e009986.

27. Warshak CR, Regan J, Moore B, Magner K, Kritzer S, Van Hook J. Association between marijuana use and adverse obstetrical and neonatal outcomes. J Perinatol 2015;35:991-5.

28. Bolhuis K, Kushner SA, Yalniz S, Hillegers MH, Jaddoe VW, Tiemeier $\mathrm{H}$, et al. Maternal and paternal cannabis use during pregnancy and the risk of psychotic-like experiences in the offspring. Schizophr Res 2018;202:322-7.

29. Goldschmidt L, Day NL, Richardson GA. Effects of prenatal marijuana exposure on child behavior problems at age 10. Neurotoxicol Teratol 2000;22:325-36.

30. Leech SL, Larkby CA, Day R, Day NL. Predictors and correlates of high levels of depression and anxiety symptoms among children at age 10. J Am Acad Child Adolesc Psychiatry 2006;45:223-30.

31. Fong GT, Elton-Marshall T, Driezen P, Kaufman AR, Cummings KM, Choi K, et al. U.S. adult perceptions of the harmfulness of tobacco products: descriptive findings from the 2013-14 baseline wave 1 of the path study. Addict Behav 2019;91:180-7.
32. Baeza-Loya S, Viswanath H, Carter A, Molfese DL, Velasquez KM, Baldwin PR, et al. Perceptions about e-cigarette safety may lead to e-smoking during pregnancy. Bull Menninger Clin 2015;78:243-52.

33. Pearson JL, Richardson A, Niaura R, Vallone DM, Abrams DB. E-cigarette awareness, use, and harm perceptions in US adults. Am J Pub Health 2012;102:1758-66.

34. Wagner NJ, Camerota M, Propper C. Prevalence and perceptions of electronic cigarette use during pregnancy. Matern Child Health J 2017;21:1655-61.

35. Garbutt JM, Miller W, Dodd S, Bobenhouse N, Sterkel R, Strunk RC. Parental use of electronic cigarettes. Acad Pediatr 2015;15:599-604.

36. Spackman E, Haines-Saah R, Danthurebandara VM, Dowsett LE, Noseworth T, Clement FM. Marijuana use and perceptions of risk and harm: a survey among Canadians in 2016. Healthc Policy 2017;13:17-27.

37. Hughes A, Lipari RN, Williams MR. Marijuana use and perceived risk of harm from marijuana use varies within and across states. The CBHSQ Report. Rockville, MD: Substance Abuse and Mental Health Services Administration (US); 2013-2016 Jul 26.

38. Romijnders KA, Van Osch L, De Vries H, Reiskje T. Perceptions and reasons regarding e-cigarette use among users and non-users: a narrative literature review. Int J Environ Res Public Health 2018;15:1190.

39. Ng DM, Jeffery RW. Relationships between perceived stress and health behaviors in a sample of working adults. Health Psychol 2003;22:638-42.

40. McCormick MC, Brooks-Gunn J, Shorter T, Holmes JH, Wallace CY, Heagarty MC. Factors associated with smoking in low-income pregnant women: relationship to birth weight, stressful life events, social support, health behaviors and mental distress. J Clin Epidemiol 1990;43:441-8.

41. Pregnancy Risk Assessment Monitoring System, Phase 7. Bethesda, MD: Center for Disease Control and Prevention; 2012 Jan 30.

42. United States Department of Health and Human Services. National Institutes of Health. National Institute on Drug Abuse, and United States Department of Health and Human Services. Food and Drug Administration. Center for Tobacco Products. Population Assessment of Tobacco and Health (PATH) Study [United States]. Ann Arbor, MI: Inter-university Consortium for Political and Social Research; 2019-04-08. https://doi. org/10.3886/ICPSR36231.v18.

43. Centers for Disease Control and Prevention (CDC). National Center for Health Statistics (NCHS). National Health and Nutrition Examination Survey Questionnaire (or Examination Protocol, or Laboratory Protocol). Hyattsville, MD: U.S. Department of Health and Human Services, Centers for Disease Control and Prevention; 2013-2014.

44. US Census Bureau. Race [Internet]. US census bureau [updated Jan 23, 2018]. Available from: https://www.census.gov/topics/ population/race/about.html.

45. Rodu B, Plurphanswat N. E-cigarette use among US adults: population assessment of tobacco and health (PATH) study. Nicotine Tob Res 2018;20:940-8.

46. Glover LM, Ma JZ, Kesh A, Tompkins LK, Hart JL, Mattingly DT, et al. The social patterning of electronic nicotine delivery system use among US adults. Prev Med 2018;116:27-31.

47. Crume TL, Juhl AL, Brooks-Russell A, Hall KE, Wymore E, Borgelt LM. Cannabis use during the perinatal period in a state with 
legalized recreational and medical marijuana: the association between maternal characteristics, breastfeeding patterns, and neonatal outcomes. J Pediatr 2018;197:90-6.

48. Ryan SA, Ammerman SD, O'Connor ME, Committee on Substance Use and Prevention, Section on Breastfeeding. Marijuana use during pregnancy and breastfeeding: implications for neonatal and childhood outcomes. Pediatrics 2018;142:e20181889.

49. American College of Obstetricians and Gynecologists Committee on Health Care for Underserved Women; ACOG Committee on Obstetric practice. ACOG committee opinion. Number 721, October 2017. Smoking cessation during pregnancy. Obstet Gynecol 2017;130:200-4.

50. Farber HJ, Walley SC, Groner JA, Nelson KE, Section on Tobacco Control. Clinical practice policy to protect children from tobacco, nicotine, and tobacco smoke. Pediatrics 2015;136:1008-17.

51. Tong VT, Dietz PM, Morrow B, D’Angelo DV, Farr SL, Rockhill KM, et al. Trends in smoking before, during, and after pregnancy - pregnancy risk assessment monitoring system, United States, 40 Sites, 2000-2010. MMWR Surveill Summ 2013;62:1-19.

52. Reitan T, Callinana S. Changes in smoking rates among pregnant women and the general female population in Australia, Finland, Norway, and Sweden. Nicotine Tob Res 2017;19:282-9.
53. Ko JY, Farr SL, Tong VT, Creanaga AA, Callaghan WM. Prevalence and patterns of marijuana use among pregnancy and non-pregnant women of reproductive age. Am J Obstet Gynecol 2015;213:201.

54. Curtin SC, Matthews TJ. Smoking prevalence and cessation before and during pregnancy: data from the birth certificate, 2014. Natl Vital Stat Rep 2016;65:1-14.

55. Caraballo RS, Shafer PR, Patel D, Davis KC, McAfee TA. Quit methods used by US adult cigarette smokers, 2014-2016. Prev Chronic Dis 2017;14:E32.

56. Borelli B, O’Connor GT. E-cigarettes to assist with smoking cessation. N Engl J Med 2019:678-9.

57. Morton SM, Bandara DK, Robinson EM, Carr PE. In the 21st century, what is an acceptable response rate? Aust N Z J Public Health 2012;36:106-8.

58. Chung EK, Nurmohamed L, Mathew L, Elo IT, Coyne JC, Culhane JF. Risky health behaviors among mothers-to-be: the impact of adverse childhood experiences. Acad Pediatr 2010;10:245-51.

Supplementary Material: The online version of this article offers supplementary material (https://doi.org/10.1515/jpm-2019-0392). 